\title{
Inter- and Intrachain Associations of an Ethylene-Vinyl Acetate Random Copolymer in Dilute 1,2-Dichloroethane Solutions
}

\author{
J in Wen Qian, ,,t Xiaohui Wang, ${ }^{\ddagger}$ Guo Rong Qi, ${ }^{\dagger}$ and Chi Wu,,
}

\author{
Department of Polymer Science and Enginering, Zhejiang University, \\ Hangzou, 310027, China, and Department of Chemistry, The Chinese \\ University of Hong Kong, Shatin, Hong Kong
}

Received October 22, 1996; Revised Manuscript Received March 20, $1997^{\otimes}$

\begin{abstract}
Laser light scattering (LLS) has been used to study an ethylene-vinyl acetate (EVA) random copolymer in 1,2-dichlorothane. The EVA solution was treated using two different thermal processes: (1) quickly quenching from $70{ }^{\circ} \mathrm{C}$ to $0{ }^{\circ} \mathrm{C}$; and (2) slowly cooling from $70{ }^{\circ} \mathrm{C}$ to room temperature and annealing at room temperature for $24 \mathrm{~h}$ before further quenching to $0{ }^{\circ} \mathrm{C}$. The LLS results reveal the formation of larger EVA aggregates in both of the thermal processes. After the EVA solution was reheated from $0{ }^{\circ} \mathrm{C}$ to a higher temperature, we found that the size of the EVA aggregates formed in the fast cooling process is nearly independent of temperature in the range of $15-30^{\circ} \mathrm{C}$, but the size of the EVA aggregates formed in the slow cooling process increases and reaches a maximum in the same temperature range. In both of the cases, the EVA aggregates dissociate at higher temperatures. The formation kinetics of the EVA aggregates at $40^{\circ} \mathrm{C}$ and the effect of the annealing time at room temperature were also investigated. All results lead us to realize that in the fast cooling process the EVA aggregates were formed mostly through the aggregation (packing) of individual EVA collapsed chains (intrachain aggregation), while in the slow cooling process, the EVA aggregates are microgel-like particles formed through the winding of the EVA chains (interchain association). We also found that there is a good correlation between the size of the EVA aggregates and the pour point of a wax solution doped with EVA.
\end{abstract}

\section{Introduction}

It is known that ethylene-vinyl acetate (EVA) copolymers have a broad range of applications, such as in adhesives, coatings, and additives. ${ }^{1}$ Recently, much attention has been paid to the applications of using EVA copolymers and their derivatives to improve the flowing of crude oil, diesel, and other base oils at low temperature, ${ }^{2-4}$ such as in depressing the pour point and reducing the oil viscosity. It has been suggested that the ability of improving the flowing properties of oils might be related to the polymer chain dimension of the pour-point depressant in solution and also to the thermal history of the oil after doped with the pourpoint depressant. ${ }^{5-8}$ However, there is no direct microscopic evidence to link the improvement of the flowing behavior of oil to microscopic molecular parameters of the pour-point depressant doped in the oil. Previously, we have used viscometry to study the concentration and temperature dependence of the association of the EVA copolymers in different solvents, such as 1,2-dichloroethane, cyclohexane, xylene, and chlor oform. ${ }^{9}$ We found that the EVA chains can form a stronger association in the 1,2-dichloroethane at low temperature in comparison with in other solvents, but viscosity cannot directly provide us microscopic information. In this study, both static and dynamic laser light scattering (LLS) were used to investigate an ethylene-vinyl acetate random copolymer in 1,2-dichloroethane because LLS as a well-established method has been widely used to study the chain dynamics of macromolecules and the association of block copolymers in solution. ${ }^{10-13}$ We intend to find the status of the EVA chains in 1,2-dichloroethane after the EVA solution was treated using different thermal processes. We also wish

\footnotetext{
* To whom correspondence should be addressed.

† Zhejiang University.

\# The Chinese University of Hong Kong.

${ }^{\otimes}$ Abstract published in Advance ACS Abstracts, May 1, 1997.
}

to establish a correlation between the solution properties of EVA and the flowing behavior of the oil doped with EVA. The ultimate objective of this study is to have a better understanding of the fundamentals re lated to the polymer chain dimension in oils, so that we will be able to choose and design a better pour-point depressant.

\section{Experimental Section}

Sample. The ethylene-vinyl acetate (EVA) sample used was a fraction of a commercial EAV product from the Chemical Engineering Institute of Shanghai. Its number-average mol ar mass $\left(2.97 \times 10^{4}\right)$, polydispersity index (1.63), and vinyl acetate content (30.8\%) were respectively determined using a Knauer membrane osmometer, Waters 150 size excluded chromatography (SEC), and a Carlo Erta-1106 element analyzer. First, the EVA sample was dissolved in 1,2-dichloroethane at $45^{\circ} \mathrm{C}$, and then the solution was heated to $\sim 60^{\circ} \mathrm{C}$ and clarified directly into a dust-free LLS cell by $0.22 \mu \mathrm{m}$ Millipore filter. The solution after the clarification was sealed and heated to $70^{\circ} \mathrm{C}$ and maintained at $70^{\circ} \mathrm{C}$ for at least $1 \mathrm{~h}$ to insure a complete dissociation of the EVA chains. The solution was then respectively treated using fast and slow cooling processes. In the fast process, the EVA solution was quickly quenched from 70 to $0{ }^{\circ} \mathrm{C}$ and kept at $0{ }^{\circ} \mathrm{C}$ for 30 min before being reheated to a higher measurement temperature, while in the slow process, the EVA solution was slowly cooled from $70^{\circ} \mathrm{C}$ to room temperature by switching off the heating bath and annealed at room temper ature for $24 \mathrm{~h}$ before a final quenching from room temperature to $0{ }^{\circ} \mathrm{C}$. Except as stated otherwise, the measurements were conducted after equilibration at each temperature.

Laser Light Scattering (LLS). A modified commercial LLS spectrometer (ALV/SP-125) equipped with a multi- $\tau$ digital time correlation (ALV5000) and a solid-state laser (ADLAS DPY 425II, output power $=400 \mathrm{mw}$ at $\lambda_{0}=532 \mathrm{~nm}$ ) as light source was used. The incident beam was vertically polarized with respect to the scattering plane. The detail of LLS instrumentation and theory can be found el sewhere. ${ }^{14-16}$ In dynamic LLS, the measured time correlation functions were 


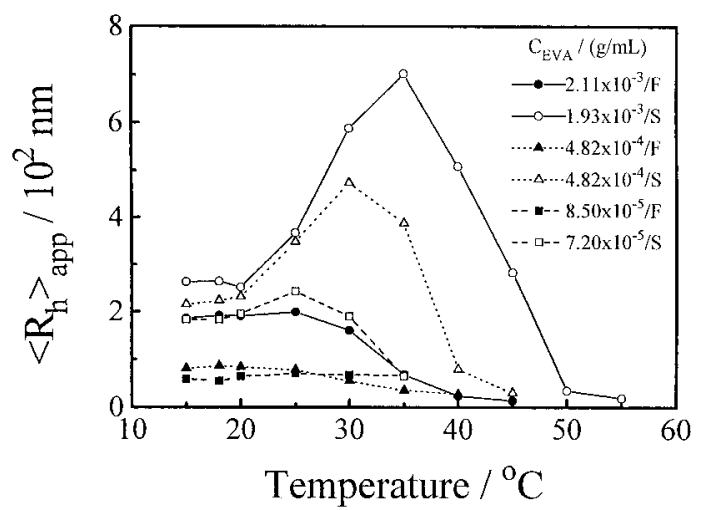

Figure 1. Typical temperature dependence of the apparent average hydrodynamic radius $\left\langle R_{h}\right\rangle_{\text {app }}$ of the EVA aggregates in 1,2-dichlorothane, where the filled and hollow symbols, respectively, represent the EVA solution treated using the fast and slow cooling processes. The subscript "app" indicates that the values of $\left\langle R_{h}\right\rangle$ were obtained at a finite scattering angle of $30^{\circ}$.

analyzed by both the Cumulants and Laplace inversion (CONTIN) programs equipped with the correlator to obtain the average line width $(\langle\Gamma\rangle)$ and line-width distribution $(G(\Gamma))$. $\Gamma$ can be related to the translational diffusion coefficient (D) by $\Gamma=\mathrm{Dq}^{2}$, where $\mathrm{q}=4 \pi \mathrm{n} \sin (\theta / 2) / \lambda$ with $\mathrm{n}, \theta$, and $\lambda$ being the solvent refractive index, the scattering angle, and the wavelength of light in vacuum, respectively. $D$ is further related to the hydrodynamic radius $R_{h}$ by the Stokes-Einstein equation, $\mathrm{R}_{\mathrm{h}}=\mathrm{k}_{\mathrm{B}} \mathrm{T} /(6 \pi \eta \mathrm{D})$, with $\mathrm{k}_{\mathrm{B}}, \mathrm{T}$ and $\eta$ being the Bol tzmann constant, temperature $(K)$, and the solvent viscosity, respectively. Therefore, $G(D)$ can be easily converted to the hydrodynamic radius distribution $\left(f\left(R_{h}\right)\right)$. The scattering angle was kept at $30^{\circ}$ for all measurements because in this study we are only interested in the relative change of the average hydrodynamic radius $\left(\left\langle R_{h}\right\rangle=\int_{0}^{\infty} f\left(R_{h}\right) d R_{h}\right)$ and $f\left(R_{h}\right)$.

\section{Results and Discussion}

Figure 1 shows the typical temperature dependence of the apparent average hydrodynamic radius $\left\langle R_{h}\right\rangle_{\text {app }}$ of the EVA aggregates in 1,2-dichloroethane, where the filled and hollow symbols, respectively, represent the EVA solution treated using the fast and slow cooling processes. As stated before, after cooling the sample to $0{ }^{\circ} \mathrm{C}$, we reheated the sample to the measured temperature and waited for $\sim 30 \mathrm{~min}$ to make sure that the sample reached a temperature equilibrium. Each heating step took $\sim 1 \mathrm{~h}$. It should be stated that the processes are practically reproducible. The subscript "app" indicates that the values of $\left\langle R_{h}\right\rangle$ were obtained at a finite scattering angle of $30^{\circ}$. F or the EVA aggregates formed in the slow cooling process, $\left\langle\mathrm{R}_{\mathrm{h}}\right\rangle_{\text {app }}$ gradually increases to a maximum as temperature increases in the range $15-30{ }^{\circ} \mathrm{C}$, while for the EVA aggregates formed in the fast cooling process $\left\langle R_{h}\right\rangle_{a p p}$ is nearly independent of temperature in the same temperature range. In both of these cases, $\left\langle R_{h}\right\rangle_{\text {app }}$ starts to decrease at $\sim 30{ }^{\circ} \mathrm{C}$ and approaches a similar value at $\sim 50{ }^{\circ} \mathrm{C}$, reflecting the dissolution of the EVA aggregates. It should be stated that at $15^{\circ} \mathrm{C}$ the EVA aggregates are very stable. Figure 1 shows that for a similar EVA concentration the size of the EVA aggregates formed in the slow cooling process is larger than that formed in the fast cool ing process. On the other hand, for a given cooling process, the size of the EVA aggregates formed in a higher EVA concentration is larger than that formed in a lower EVA concentration, which is understandable because when the EVA concentration is higher there are more chances for individual EVA chains to aggregate with each other.

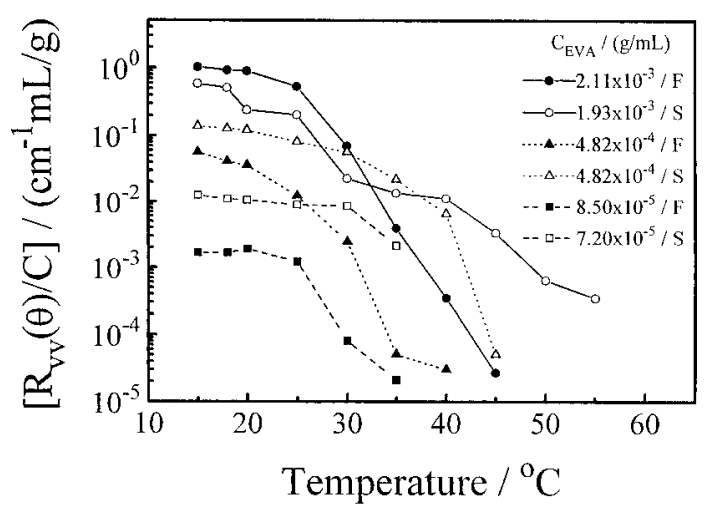

Figure 2. Temperature dependence of specific Rayleigh ratio $\mathrm{R}_{\mathrm{vv}}(\theta) / C$ of three EVA solutions after being treated using the two different thermal processes, where $R_{v v}(\theta)$ is the Rayleigh ratio, $\theta$ is $30^{\circ}$, and $C$ is the $E V A$ concentration.

Schematic of the dissolution of the EVA aggregates
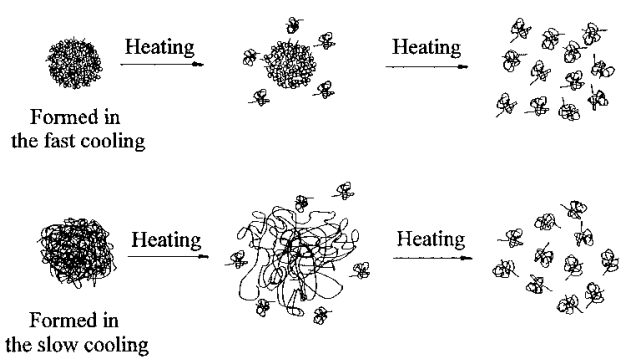

Figure 3. Schematic of the dissolution of two types of the EVA aggregation respectively formed in the two different thermal processes.

Figure 2 shows the temperature dependence of specific Rayleigh ratio $\mathrm{R}_{\mathrm{vv}}(\theta) / C$ of threeEVA solutions after being treated using the two different thermal processes, where $\mathrm{R}_{\mathrm{vv}}(\theta)$ is the Rayleigh ratio, $\theta$ is $30^{\circ}$ and $C$ is the EVA concentration. It is known that $R_{v v}(\theta) / C$ is proportional to the weight-average molar mass $\left(M_{w}\right)$, i.e., $\mathrm{R}_{\mathrm{wv}}(\theta) / C \propto \mathrm{M}_{\mathrm{w}}$. Figure 2 clearly shows that $\mathrm{R}_{\mathrm{vv}}(\theta) / \mathrm{C}$ decreases as temperature increases in spite of the fact that the EVA solutions were treated by the two different thermal processes. The temperature dependence of $R_{w v}(\theta) / C$ is quite different from that of $\left\langle R_{h}\right\rangle_{\text {app }}$ shown in Figure 1. The decrease of $R_{v v}(\theta) / C$, i.e., the decrease of $M_{w}$ of the EVA aggregates, indicates the dissolution of the EVA aggregates. It is interesting to note that for the EVA sol utions treated using the fast cooling process the decrease of $R_{v v}(\theta) / C$ starts at a lower temperature and the decreasing rate is fast, implying that the dissolution of the EVA aggregates formed in the fast cooling process is easier, or in other words, the interchain association formed in the fast cooling process is weaker than that formed in the slow cooling process.

A combination of Figures 1 and 2 leads us to realize two different kinds of aggregation of the EVA chains, which is schematically shown in Figure 3 . In the fast cooling process, individual EVA chains have less chances to aggregate together before they became individual collapsed chains; namely, in the fast cooling process, the aggregation is dominated by the intrachain association. In this case, the final EVA aggregates are formed mainly through the "packing" of the collapsed EVA chains; however, in the slow cooling process, the EVA chains have more time to wind with each other by the interchain association to form microgel-like particles in the solution. During the reheating process, 1,2-dichloroethane gradually becomes a better solvent for EVA so that two simultaneous processes, namely the swelling 

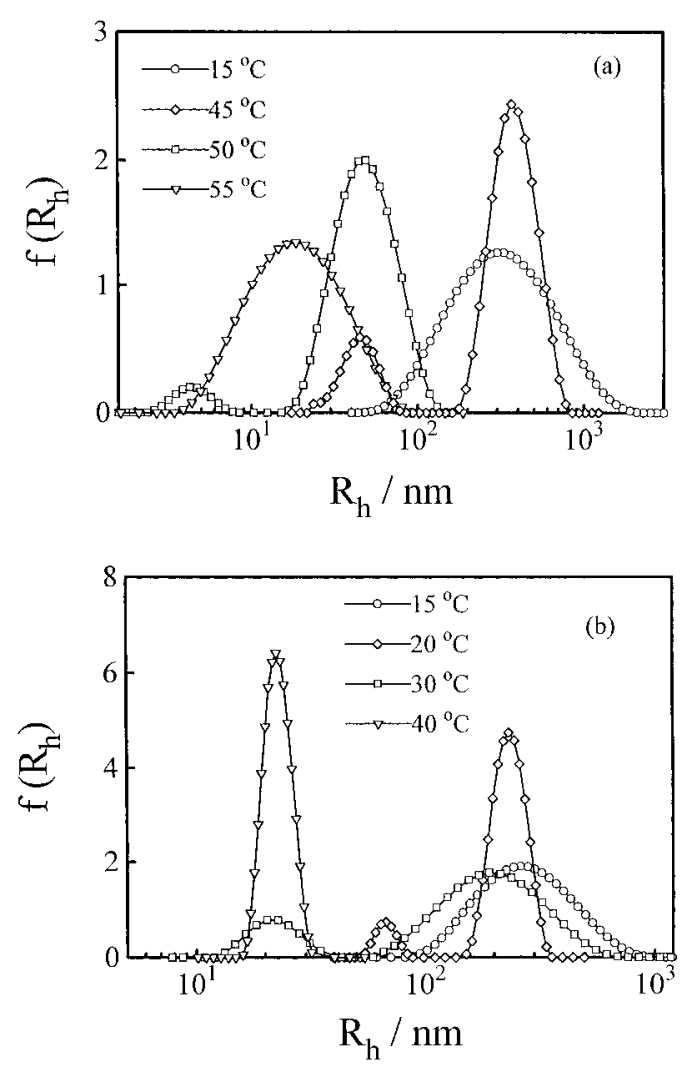

Figure 4. Typical hydrodynamic radius distributions of the EVA aggregates. Parts $a$ and $b$ respectively represent the EVA aggregates formed in the slow and fast cooling processes, where the EVA concentration is $\sim 2 \times 10^{-3} \mathrm{~g} / \mathrm{mL}$.

and dissociation of the EVA aggregates, are expected. For the EVA aggregates formed through the "packing" of individual collapsed EVA chains in the fast cooling process, the dissociation should be faster than the swelling because there is no strong interchain association between the collapsed EVA chains. This is why $\left\langle\mathrm{R}_{\mathrm{h}}\right\rangle_{\text {app }}$ decreases as temperature increases. On the other hand, for the EVA aggregates formed through the winding of the EVA chains in the slow cooling process, the microgel-like EVA particle has to swell before its dissociation. This explains the initial increase of $\left\langle R_{h}\right\rangle_{\text {app }}$ shown in Figure 1. In both of the cases, the dissociation leads to the decrease of the molar mass of the EVA aggregates, resulting in the decrease of $R_{v v}(\theta) / C$ shown in Figure 2. It is not difficult to realize that the dissociation of the EVA aggregates formed through the packing of the collapsed EVA chains is faster and easier than that consisting of the wound EVA chains. It is worth noting that the solutions are not in a state of thermodynamic equilibrium; namely, if the solution is annealed at high temperatures for a sufficiently long time, they will reach the same state in spite of different thermal histories.

Parts $a$ and $b$ of Figure 4 show typical hydrodynamic radius distributions of the EVA aggregates after the EVA solution was respectively treated by the slow and fast cooling processes and then reheated from $0{ }^{\circ} \mathrm{C}$ to different measurement temperatures. It clearly shows that, as temperature increases, the EVA aggregates become smaller and smaller. Simultaneously, the second peak located at the smaller $R_{h}$ side appears in $f\left(R_{h}\right)$, indicating that parts of the EVA aggregates have disintegrated. At an even higher temperature, all EVA aggregates have dissociated into small fragments and even individual EVA chains. It should be noted that

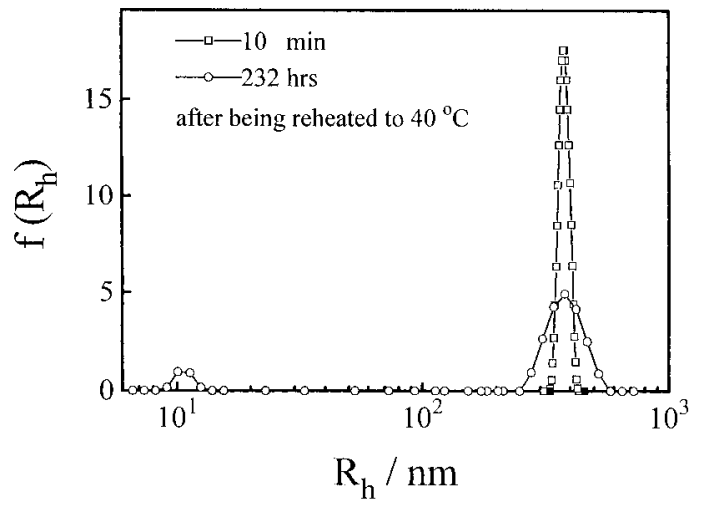

Figure 5. Typical time dependence of the hydrodynamic radius distribution of the EVA aggregates after the EVA solution was treated by the slow cooling process and then reheated from 0 to $40^{\circ} \mathrm{C}$.

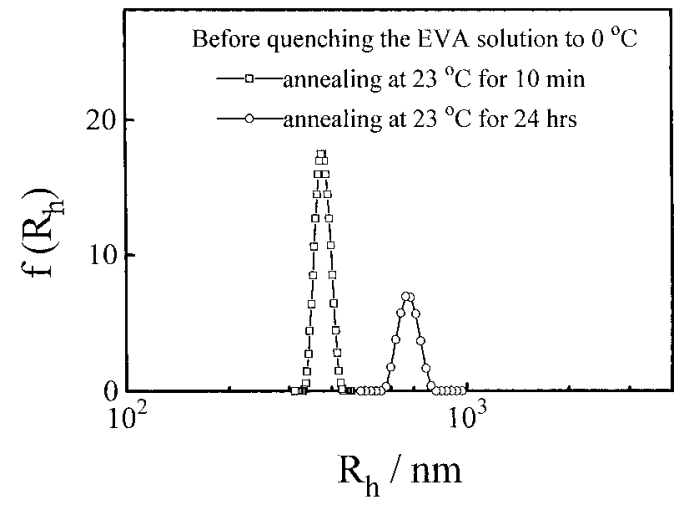

Figure 6. Hydrodynamic radius distribution of the EVA aggregates formed in the slow cooling process after annealing at room temperature for two different lengths of time, where $f\left(R_{h}\right) s$ were determined after the EVA solutions were reheated from 0 to $40{ }^{\circ} \mathrm{C}$.

the EVA aggregates formed in the fast cooling process dissociate at a much lower temperature, indicating a weaker intrachain association and supporting the model presented in Figure 3.

Figure 5 shows typical time dependence of the hydrodynamic radius distribution of the EVA aggregates after the EVA solution was treated by the slow cooling process and then reheated from 0 to $40{ }^{\circ} \mathrm{C}$. I nitially, the EVA aggregates were very narrowly distributed with only one peak. After the solution was allowed to stand at $40{ }^{\circ} \mathrm{C}$ for $232 \mathrm{~h}$, the peak became broader and a second peak located at $\sim 10 \mathrm{~nm}$ appeared in $f\left(R_{h}\right)$. The broadening of the first peak indicates the swelling of the EVA aggregates in the solution, and the appearance of the second peak shows the dissolution of the EVA aggregates at $40{ }^{\circ} \mathrm{C}$. Figure 5 also clearly shows that the result presented in Figures 1 and 2 at each temperature should be very slowly dependent on time.

Figure 6 shows the annealing time dependence of the hydrodynamic radius distribution of the EVA aggregates formed in the slow cooling process, where $f\left(R_{h}\right) s$ were measured after the EVA solutions were reheated from 0 to $40{ }^{\circ} \mathrm{C}$. As expected, a longer annealing time leads to larger EVA aggregates because when the EVA solution was annealed at $23{ }^{\circ} \mathrm{C}$ for a longer time the EVA chains and aggregates had more chances to combine with each other to form larger EVA particles. On the other hand, a longer annealing time also allows the development of more and stronger physical crosslinking between different EVA chains. Figure 6 further indicates that in the slow cooling process the aggrega- 


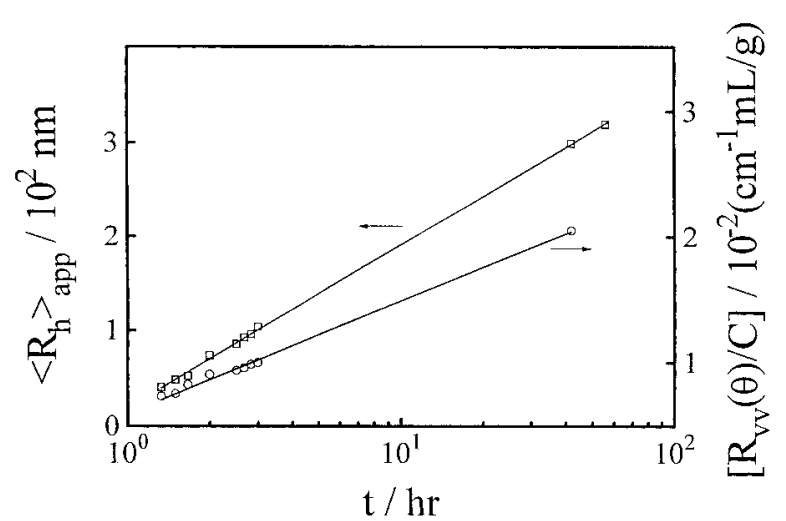

Figure 7. Aggregation kinetics of the EVA chains after the EVA solution was sl owly cooled from 70 to $40{ }^{\circ} \mathrm{C}$, where $\left\langle R_{h}\right\rangle_{\text {app }}$ is the apparent average hydrodynamic radius, $R_{v v}(\theta)$ is the Rayleigh ratio, and C is the EVA concentration. The two lines respectively represent the least square fittings of $\left\langle R_{h}\right\rangle_{\text {app }}=64.6$ $+129 \log (\mathrm{t})$ and $\mathrm{R}_{\mathrm{vv}}(\theta) / \mathrm{C}=6.01 \times 10^{-3}+8.86 \times 10^{-3} \log (\mathrm{t})$.

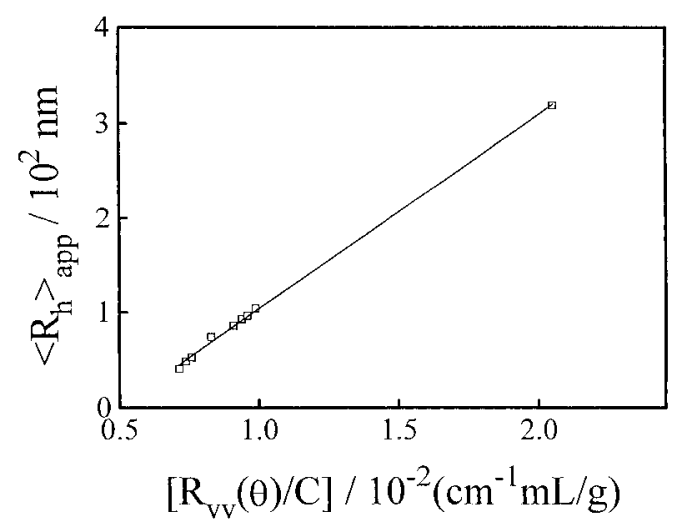

Figure 8. Plot of $\left\langle R_{h}\right\rangle_{a p p} v s R_{v v}(\theta) / C$, when the line represents a least-squares fitting of $\left\langle\mathrm{R}_{\mathrm{h}}\right\rangle=-101+2.05 \times 10^{4} \mathrm{R}_{\mathrm{vv}}(\theta) / \mathrm{C}$.

tion of the EVA chains is mainly through the interchain association.

Figure 7 shows the aggregation kinetics of the EVA chains after the EVA solution was slowly cooled from 70 to $40^{\circ} \mathrm{C}$. It is interesting to see that both $\left\langle\mathrm{R}_{\mathrm{h}}\right\rangle_{\text {app }}$ and $R_{w}(\theta) / C$ are proportional to log(t), for which we have no explanation at this moment. These two linear relations lead to $\left\langle R_{h}\right\rangle_{\text {app }} \propto R_{v v}(\theta) / C$ shown in Figure 8 . Since $R_{v v}(\theta) / C \propto M_{w}$, Figure 8 actually indicates that $M_{w} \propto\left\langle R_{h}\right\rangle_{a p p}$, namely the dimension of the EVA aggregates linearly increases as the mass increases, suggesting a linear aggregation of the EVA chains when the EVA solution was slowly cooled from 70 to $40^{\circ} \mathrm{C}$, or in other words, the EVA aggregates formed in the initial stage and at a higher temperature are fibrouslike.

So far we have demonstrated that by controlling the cooling rate, the annealing time and the temperature at which the EVA aggregates are formed, the size of the EVA aggregates can be varied. The next important question is how the size of the EVA aggregates can be correlated to the flowing properties of the oil doped with EVA. Figure 9 shows the solvent composition dependence of the apparent average hydrodynamic radius $\left(\left\langle R_{h}\right\rangle_{\text {app }}\right)$ of the EVA aggregates and the change of the pour point $\left(\Delta T_{p p}\right)$ in the cyclohexane/1,2-dichloroethane mixture containing $10 \mathrm{w} \%$ of wax and $300 \mathrm{ppm}$ of EVA. It clearly shows that when the composition of the mixture reaches $\sim 0.6$ the addition of EVA has nearly no effect on the pour point anymore. In the composition range of $60-100 \%$, the values of $\left\langle\mathrm{R}_{\mathrm{h}}\right\rangle_{\text {app }}$ reveal that most

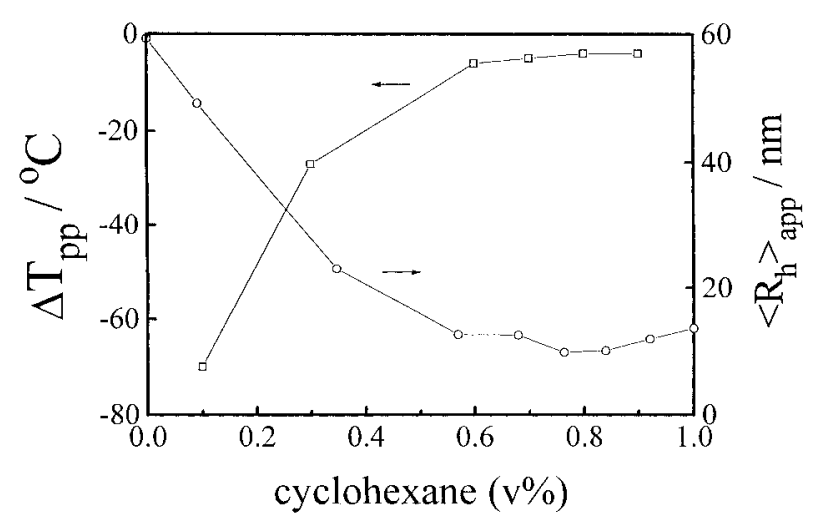

Figure 9. Solvent composition dependence of the apparent average hydrodynamic radius $\left(\left\langle R_{h}\right\rangle_{a p p}\right)$ of the EVA aggregates and the change of the pour point $\left(\Delta T_{p p}\right)$ in the cyclohexane/ 1,2-dichloroethane mixture containing 10 wt \% of wax and 300 ppm of EVA.

of the EVA chains exist as individual chains in the solvent mixture. Figure 9 also shows that the larger the EVA aggregates, the larger the decrease of the pour point. It is interesting to find that the macroscopic decrease of the pour point of an oil is related to the microscopic association of the EVA chains in the solvent mixture.

\section{Conclusions}

This study shows that the intrachain and interchain associations of ethylene-vinyl acetate (EVA) in 1,2dichloroethane can be greatly influenced by the thermal process; namely, in a fast cooling process, the EVA aggregates are formed mainly through the "packing" of individual collapsed chains, while, in a slow cooling process, the EVA aggregates are mi crogel-like particles formed mainly through the winding of the EVA chains and fibrous-like aggregates. The particle size of the EVA aggregates can be control led by varying the initial EVA concentration, the annealing time at room temperature and the cool ing rate. We al so showed that the decrease of the macroscopic pour point of a wax solution can be directly related to the microscopic size of the EVA aggregates in the solution. By controlling the particle size, we can vary the pour point or, in a more broad meaning, the flowing properties of a wax solution.

Acknowledgment. The financial support of this work by the RGC (the Research Grants Council of Hong Kong Government) Earmarked Grant 1995/96 (CUHK 453/95P, A/C No. 2160046) is gratefully acknowledged. J.W.Q. and G.R.Q. al so would like to acknowledge the financial support of this work by the National Nature Science Foundation of China.

\section{References and Notes}

(1) Salyer, I. O.; Kenyon, A. S. J . Polym. Sci. A-1 1971, 9, 3083.

(2) Gilby, G. W. Proceedings of the 2nd International Conference on Chemicals in the Oil Industry; Royal Society of Chemistry: London, 1983; Vol. 45, p 108.

(3) Sunil Kumar, M. N. The Institute of Petroleum, Quarterly J ournal of Technical Papers 1989, Oct.-Dec., 47.

(4) Qian, J. W.; Qi, G. R.; Ding, X. Z.; Yang, S. L. Fuel 1996, 3, 307.

(5) Qian, J. W.; Qi, G. R.; Han, D. L.; Yang, S. L. Fue 1996, 2, 163.

(6) Qian, J. W.; Qi, G. R.; Xu, Y. L.; Yang, S. L. J . Appl. Polym. Sci. 1996, 60, 1575.

(7) Liu, Q. L.; Quan, Z. Y. Acta Pet. Sin. 1986, 7, 119.

(8) Song, J. X.; Huang, Z. T.; Pan, D. R. Acta Pet. Sin. 1989, 10, 115. 
(9) Qian, J . W.; Qi, G. R.; Cheng, R. S. Eur. Polym. J ., in press. (10) Mandema, W.; Zeldenrust, H.; Emeis, C. A. Makromol. Chem. 1979, 180, 1521.

(11) Mandema, W.; Emeis, C. A.; Zeldenrust, H. Makromol . Chem. 1979, 180, 2163.

(12) Quintana, J . R.; J anez, M. D.; Katime, Z. Polymer 1996, 37, 3531.

(13) Zhang, Y.; Wu, C.; Fang.; Q.; Zhang, Y. X. Macromolecules 1996, 29, 2494.
(14) Chu, B. Laser Light Scattering; Academic Press: New York 1974.

(15) Pecora, R. Dynamic Light Scattering; Plenum Press: New York, 1976.

(16) Wu, C.; Siddiq, M.; Woo, K. F. Macromolecules 1995, 28, 4914. MA961560E 